\title{
Participation of Multi-Terminal HVDC Grids in Frequency Regulation Services
}

\author{
C. L. Moreira, J. R. Gouveia, B. Silva \\ INESC TEC - INESC Technology \& Science \\ CPES - Centre for Power and Energy Systems \\ Rua Dr. Roberto Frias, 378, 4200-465 Porto, Portugal \\ carlos.moreira@inescporto.pt
}

\begin{abstract}
This paper addresses the provision of frequency control services with multi-terminal HVDC grids interconnecting several asynchronous $\mathrm{AC}$ control areas and integrating offshore wind farms. Regarding the operational performance of the multiterminal HVDC grid, it is discussed and proposed a communication-free regulation scheme that allows these type of infrastructures to actively participate in primary frequency regulation services and provision of inertial emulation capabilities among the non-synchronous areas. Additionally, the proposed control scheme is extended such that offshore wind generators can also actively provide inertia and primary frequency control to the mainland AC grid. The main rational of the proposed control scheme relies of a cascading control mechanism based on the modulation of active power as a function of the frequency in the HVDC converter stations connected to mainland AC grids and on the control of the frequency in the HVDC converters associated to offshore wind farms. The DC grid voltage variations resulting from this principle is used as a natural communication channel to develop the control loops to be used in all the converter stations. The effectiveness of the proposed strategy is illustrated in the case of two non-synchronous areas linked by a multi-terminal HVDC system connecting two offshore wind farms.
\end{abstract}

Keywords-Frequency control, HVDC power transmission, Inertial emulation, Multi-terminal HVDC system, Offshore wind farms

\section{INTRODUCTION}

The development of additional electrical interconnections is presently being planned by sea exploiting offshore grids, as it is documented in the European project "TWENTIES" and in the Intelligent Energy Europe projects "OffshoreGrid" and "TradeWind" [1-4]. In both cases, offshore interconnections are expected to provide two main requirements: the support to power exchange between AC areas and offshore wind power integration. Voltage Source Converter High Voltage Direct Current (VSC-HVDC) technology seems to be the most promising solution for the development of offshore DC connections. Regarding HVDC transmission, Multi-Terminal DC (MTDC) grids are foreseen as an alternative solution to point-to-point connections, providing higher flexibility, increased redundancy and reduction of maximum power not supplied to onshore grids in case of DC disturbances [2-3].

Conventional AC grids rely on several control levels to provide adequate power regulation while maintaining global system stability. In particular, inertial, primary, secondary and tertiary responses are responsible for dictating the global power system balance within the grid. In the moments subsequent to a sudden imbalance, inherent inertial response form synchronous units limits the rate of change of frequency, while primary control stabilizes the frequency decay through the proper adjustment of prime movers using a droop control having as input the grid frequency error.

Unlike conventional power plants equipped with synchronous generators, both HVDC interconnections between $\mathrm{AC}$ systems as well as the new renewable energy sources such as wind power lack the inherent ability to contribute for inertial emulation and primary frequency control. Additionally, largescale integration of new renewable energy such as wind power results on the displacement of conventional generation units that negatively impact the behaviour of grid frequency (leading to increased rates of change of frequency and larger absolute deviation). Therefore, system operators become more concerned regarding short term grid stability issues and there is a growing interest in requesting inertial and primary response capabilities from renewable energy sources [5-6].

In line with this requirements, some authors investigated the possibility of using wind generators to provide primary frequency control and inertial emulation capabilities [7-11]. Regarding the provision of primary frequency primary frequency control from wind generators, a common approach consists in operating them below its maximum power extraction capability (wind generator de-load), hence creating a spinning reserve margin. Additionally, the authors suggest the use of a local control loop at the wind turbine level, whose input variable is the measured frequency error that is used to turbine power output for primary frequency regulation purposes. Regarding inertial response, it is possible to obtain the desired performance relying on the extraction of kinetic energy from the rotating mass of the wind turbine.

Regarding HVDC systems, in addition to the provision of standard power flow transfer among areas, it is important to have in consideration that additional services are required in order to support system operation, namely: active contribution to frequency deviations through the adjusting the active power transmission in accordance with control parameters to be specified by the grid operators, as well as active contribution towards inertial emulation response. [12]. However, implementation of control mechanisms to achieve the desired 
performance and capabilities need to be identified and its feasibility duly demonstrated.

Since HVDC systems fully decouples AC areas to which they are connected, the direct implementation of previously referred frequency regulation functionalities is not possible. Some studies have been developed relying on communication schemes to support the desired frequency support functionality [13]. Additionally it was also investigated the possibility of endowing offshore wind farms connected to an AC grid through a point-to-point HVDC system with primary frequency control capability [14]. In order to achieve such control capabilities the offshore converter is complemented with additional control loops whose input is the AC system frequency, which is assumed to be communicated to the offshore rectifier station. However, communication-based solutions are prone to surfer from reliability and performance issues (complete loss of communication, latency or reduced data rate) which may preclude the effectiveness of such a solution. Additionally, the specificity of MTDC grids increases the challenge for the implementation of communication networks for fast control actions. In general, a communication based solution demands for some coordination regarding the decision making process for the definition and assignment of power in-feed set-points in the MTDC nodes required to control the frequency in a given AC control area. For these reasons, special attention is required in order to develop communication-independent control schemes, which rely only on local measurements. In line with this control concept, this paper presents an innovative approach consisting on the identification and development of local controllers to be installed at the VSC-HVDC converter stations which will autonomously allow the provision of frequency control services. The proposed control strategies consist on the use of a cascading reproduction of AC grid frequency deviations into MTDC voltage variations. Subsequently, MTDC voltage variations are used by the offshore VSC-HVDC for controlling the offshore wind farm AC grid frequency. Thus, offshore wind farm AC grid frequency variations will be the driving signal for frequency regulation loops to be adopted at the wind generator level.

The paper is organized as follows. In Section II, the operational framework of a MTDC system is presented, together with a discussion of its main components modelling approach. The development of the proposed control strategy for the offshore MTDC system is then discussed in Section III. Section IV presents numerical simulation results that demonstrate the effectiveness of the proposed control functionalities. Finally, Section V presents the main conclusions of the paper and proposes directions for further works.

\section{MOdeling AND Operational FramewOrK FOR Multi- TERMINAL HVDC GRIDS}

A MTDC grid provides the connection of $N$ offshore wind farms to $M$ mainland systems, as it is generically illustrated in Fig. 1. The main components of the MTDC system consist on the DC grid itself, the offshore and onshore VSC-HVDC stations and the wind generators. This section provides an overview about the simulation models used in this paper to represent the dynamics of the MTDC grid, VSC-HVDC, wind generators, AC mainland systems, as well as the control philosophy required to operate the overall system.

\section{A. Multi-terminal HVDC grid}

The DC grid is assumed to be bipolar (two cables with symmetrical voltage levels - nominal voltage $\pm V_{n}$ ). Each connection within the DC grid is performed through DC cables, which are modelled by concentrated parameter (cable resistance and inductance), according to the model introduced in [15]. The MTDC grid topology dictates the algebraic and state equations of the system.

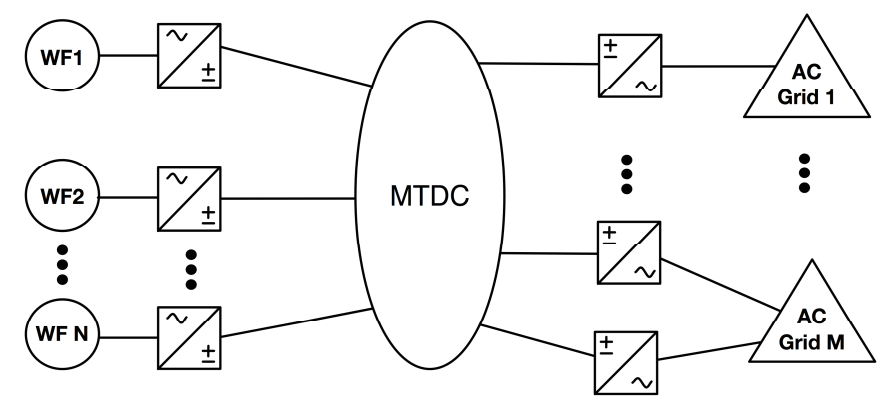

Fig. 1. Conceptual architecture of a MTDC grid.

1) Onshore converters: The onshore DC/AC converter station is responsible for the active power flow control with the mainland AC grid (and therefore for the control of the DC grid voltage in the associated terminal of the MTDC grid). As VSCHVDC converter station can independently control active and reactive power flows, two approaches can be followed regarding reactive power: reactive power injection control with a predefined power factor or AC terminal voltage control. Without lack of generality, a voltage control strategy was considered at the onshore power converter station. In terms of models implementation, the converter model was developed in the $d-q$ synchronous reference frame, as illustrated in [16-17].

2) Offshore converter: The offshore converter station acts as a controllable AC voltage source/slack bus in relation to the offshore AC grid for connecting the wind generators [16-17]. The control philosophy of this converter station consists on setting the AC-side voltage magnitude and frequency. Hence, offshore converters will extract all the available power from the offshore wind farm and inject it in the MTDC network. In terms of models implementation, the converter model was developed in the $d-q$ synchronous reference frame, as illustrated in [16-17].

\section{B. Operation and control of MTDC grids}

The general philosophy associated with the control of the MTDC grid consists on controlling DC voltage level at onshore converter stations, while the offshore converter station control the current injected into the DC grid. Therefore, appropriated DC voltage magnitudes should be maintained at each onshore converter station in order to allow proper power sharing among converters in case of power variations such as the ones occurring at the offshore wind farm level. Regarding these control issues, two approaches can be followed $[16,18]$ :

1. Maintain a constant DC voltage at a single onshore converter station, which acts as a DC slack bus, while the other offshore converter stations are controlled in order to inject a pre-defined active power into the AC grid. The main drawback of such control is related with the 
impossibility of the system to continue operation in case of losing the DC slack bus converter station.

2. Use a droop control approach in order to define the active power injection at each VSC-HVDC converter station connected to the AC grids as a function of the DC voltage deviation in relation a defined reference value. In this case, active power variations are shared among the overall onshore converter stations. In such a case, losing any converter station potentially allows the DC grid to continue its operation. Therefore, it is considered that each onshore converter sets its active power injection $P_{\text {out }}$ according to:

$$
P_{\text {out }}=P_{\text {out }}^{\text {ref }}+k_{p v}\left(V_{D C}-V_{D C}^{\text {ref }}\right)
$$

where $V_{D C}^{r e f}$ is the DC voltage reference value for a given reference power output $\left(P_{o u t}^{r e f}\right)$ and $k_{p v}$ is the active power to DC voltage droop control parameter. These parameters are assumed to be configurable and can be parameterized in the onshore converter station by an upstream supervision and control system, according to a specific operational strategy envisioned for the MTDC grid and for the onshore AC control area.

\section{B. Wind generator model}

Offshore wind generators are connected of an $\mathrm{AC}$ grid connected to the offshore HVDC-VSDC converter. The wind farm are considered to be equipped with Permanent Magnet Synchronous Generators (PMSG) that are connected to the offshore AC grid by an AC/DC/AC converter. To model the offshore wind farm, a single equivalent generator model is used, as suggested in [8].

\section{Primary Frequency Control Services In MTDC Grids}

In order to endow MTDC grids with the capability of participating in primary frequency control services to mainland $\mathrm{AC}$ control areas without relying in communication systems, it is necessary to introduce additional control loops locally at the VSC-HVDC stations. This control functions for grid frequency supporting functions can be envisioned in order to support the primary frequency control among non-synchronous areas, which can be complemented with the active participation of the offshore wind farms, it they are set with adequate reserve margins in order to participate in this type of services.

The proposed approach relies on the development of controllers that require information available only at their terminals (therefore not relying on dedicated communication channels for the time frame corresponding to the typical duration of the referred frequency regulation services). As it was previously mentioned, the use of power electronic interfaces in the MTDC grid fully decouples the different areas they interconnect. Therefore, in order to make MTDC systems to contribute to $\mathrm{AC}$ frequency regulation services, it is necessary to explicitly modulate the active power injection in a given onshore converter station as a function of the associated AC grid frequency. Consequently, the active power modulation will induce changes in the MTDC grid voltage profile, which makes possible to use it as the communication pathway for the other converter platforms in the MTDC grid. In this sense, it will be possible to exploit the MTDC grid infrastructure to coordinate and share frequency regulation functionalities among nonsynchronous areas.

Additionally, it is also possible to assume the de-load operation of offshore wind farms in order to create a proper reserve margin enabling then to provide primary reserve regulation to support the operation of mainland $\mathrm{AC}$ grids. In this case, in order to deploy the offshore wind farm reserve margin without relying on a communication infrastructure, the impact on the MTDC grid voltage resulting from the onshore VSCHVDC power modulation as a function of the $\mathrm{AC}$ grid frequency needs to be used at the offshore VSC-HVDC converter in order to control the power deployment from the wind generators. In this case, the MTDC grid voltage variations can be used in order to modulate the offshore AC grid frequency in order to make the wind generators responding to the resulting frequency variations. Thereafter, supplementary control loops can be applied at each wind generator level, similarly to the solutions used for onshore wind turbine applications regarding their participation on frequency regulation services. The additional controls that are proposed to be implemented at each offshore and onshore converter stations, as well as at wind turbine level are presented next.

\section{Advanced VSC-HVDC onshore converter control}

The onshore converter is the device responsible for interconnecting the DC network to the onshore AC system. Regarding the operation of the onshore VSC-HVDC converter station, two situations can be identified:

1. Normal operation mode: the load and generation in the onshore AC grid to which the converter station is connected are balanced, thus resulting in very small frequency deviations. In this situation, the offshore MTDC grid operates the VSC-HVDC converters in the active power/DC voltage droop control mode defined by (1) in order to assure the share offshore wind power production variations.

2. Disturbed operation mode, when the AC grid frequency to which the VSC-HVDC converter station is connected is out of a given margin (dead-band) due to any load/generation imbalance. In such situations, primary frequency regulation services are requested to be autonomously deployed in order to stabilize grid frequency.

Regarding the mode of deploying the offshore wind farm reserves, it firstly requires an additional control mechanism that translates onshore AC grid frequency grid variations to MTDC voltage profile variations. In this case, the onshore VSC-HVDC converter station measures the terminal frequency and whenever it drops below the referred margin, the active power is modulated in terms of the frequency deviation (primary response according to the $\Delta P(f)$ in equation 2 ) as well as in terms of the emulation of an inertial response through the frequency derivative and corresponding gain $k_{d}$ :

$$
P_{\text {out }}=P_{\text {out }}^{r e f}+k_{p v}\left(V_{D C}-V_{D C}^{r e f}\right)+\Delta P(f)+k_{d} \frac{d f}{d t}
$$


The power-frequency modulation included in the previous equations that represents the primary frequency control response can be defined according to the control rule presented in Fig. 2, where it is represented the maximum and minimum power deviations of the power-frequency modulation function $\left(\Delta P_{\max }\right.$ and $\Delta P_{\text {min }}$, respectively, around the actual active power operational point), as well as the grid frequency values at which they occur $\left(f_{\min }\right.$ and $f_{\max }$ respectively). The $\Delta P(f)$ function includes also a frequency dead band with a central value around the nominal frequency $f_{n}$, with a lower frequency limit to deploy the power increase $\left(f_{n}^{l}\right)$, as well as a frequency upper limit to deploy the active power decrease $\left(f_{n}^{r}\right)$.

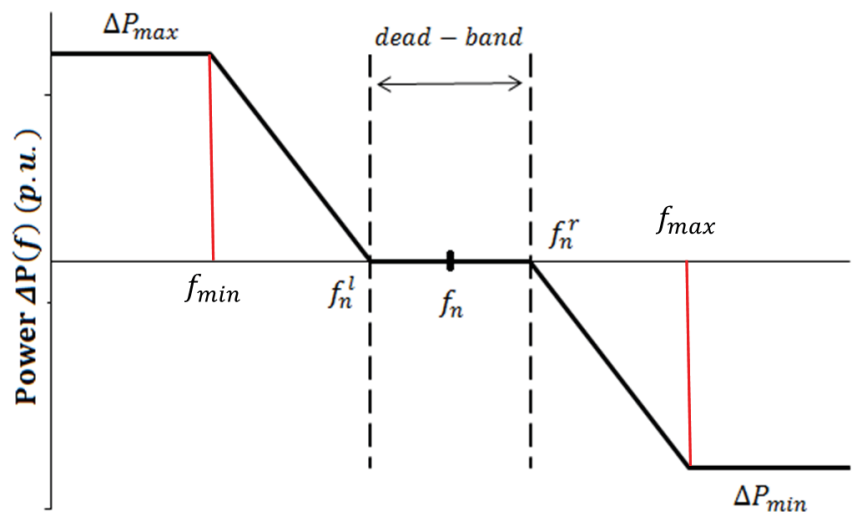

Fig. 2. Power-frequency modulation function to be include in the onshore HVDC-VSDC control station.

The proposed control allows the onshore converter stations to provide primary frequency support to onshore AC grids, as well as inertial emulation capability. The frequency droops and frequency dead-bands (in the $\Delta P(f)$ rule), as well as the derivative terms of the inertial emulation functionality can be adjusted centrally in a coordinated way by the system operators according to the specificities of the AC onshore system operating conditions.

\section{Advanced VSC-HVDC offshore converter control}

If the offshore wind farm is set to participate in the frequency regulation services, additional control functionalities need to be deployed at the offshore VSC-HVDC converter stations. From the offshore wind farm side, the VSC-HVDC converters promote the interface between the $\mathrm{AC}$ offshore wind farm grid and the MTDC grid. In order to allow offshore wind farm to provide frequency support, additional control rules must be included at the converter level. The cascading control that was previously mentioned must be used in order to modulate the offshore wind farm AC grid frequency based on the DC voltage variation at the associated MTDC grid terminal. Therefore, the offshore VSC-HVDC control should include an additional control mechanism responsible for varying the offshore AC grid frequency proportionally to the MTDC grid voltage variation. This additional control is based on a DC voltage-frequency droop that defines the new AC offshore converter frequency, as depicted in Fig. 3. The controller will evaluate the local DC voltage variation in relation to the pre-disturbance $\mathrm{DC}$ voltage value $\left(V_{D C}^{0}-V_{D C}\right)$. The DC voltage deviation passes through a dead-band which allows distinguishing the DC voltage profile variations originated by wind power variation from the onshore converter imposed DC voltage variations. The DC voltage deviation will be affected by the $K_{f}$ gain which will define the magnitude of the offshore frequency drift.

\section{E. Wind generators local controllers}

The aforementioned droop control mechanism allows the translation of onshore AC grid disturbances to offshore wind farm AC grid frequency disturbances. To provide power reserve for primary frequency regulation in wind turbines, it is considered that they are equipped with controllers using a deloading approach as proposed in $[11,12]$. Regarding the provision of primary frequency regulation and inertial emulation capabilities, the power control system of the wind generator includes additional loops responding to the offshore $\mathrm{AC}$ grid frequency deviation and to the time derivative of the offshore AC grid frequency deviations, respectively [12-15].

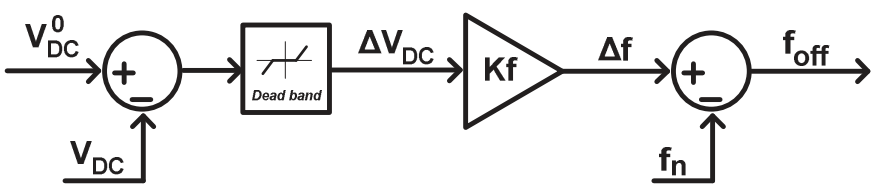

Fig. 3. Droop control mechanisms for VSC-HVDC offshore AC grid frequency.

\section{Simulation And Results}

In order to evaluate the effectiveness of the proposed control strategies to be applied at onshore and offshore converter stations, the test system shown in Fig. 4 (adapted from [19]) was used. The configuration of the MTDC grid with offshore wind farms is also shown in Fig. 5. The AC grid test system consists of two non-synchronous onshore AC areas (Area \#1 and Area $\# 2$, respectively) that are interconnected by a 4 terminal HVDC grid that also collects the power generation from two offshore wind farms (as can be seen in fig. 5). Regarding onshore AC areas, only primary frequency regulation capabilities by means of droop control are considered in the simulation results in order to better illustrate the effectiveness of the proposed control mechanisms. As it was previously mentioned, it is assumed that offshore wind farms are composed by PMSG connected to the $\mathrm{AC}$ offshore network by an AC/DC/AC converter. Each offshore wind farm was modelled by a single equivalent machine of $600 \mathrm{MW}$ of nominal power, but being operated at a power production level of $400 \mathrm{MW}$. The VSC-HVDC converters connected to the mainland grid have a nominal capacity of $800 \mathrm{MW}$ and are assumed to be injecting around 400 MW at each AC control area. The MTDC grid nominal voltage is $\pm 320 \mathrm{kV}$. A $75 \mu \mathrm{F}$ capacitor is connected to the DC side of each offshore converter station. The MTDC cable system is characterized by the following linear parameters: $\mathrm{R}=0.01 \Omega / \mathrm{km}$, $\mathrm{l}=1.4 \mathrm{mH} / \mathrm{km}$ and $\mathrm{C}=0.3 \mu \mathrm{F} / \mathrm{km}$.

The test system was fully modelled in a Matlab/Simulink simulation platform, according to the dynamic models of the components that were previously mentioned. The simulations carried out on this study are intend to demonstrate the effectiveness of the proposed control strategies and to illustrate the benefits that the possibility of endowing offshore MTDC systems with primary frequency mechanisms may have in terms 
of the onshore AC network stability. Thus, in order to contrast the AC network frequency behaviour in different situations, two major operational scenarios were considered following an onshore AC grid disturbance:

1. Scenario I: MTDC system with primary frequency control capability and inertial emulation at the onshore converter stations, being the offshore wind farm kept in operation without no regulation functionality;

2. Scenario II: MTDC system and offshore wind farm with primary frequency control capability and inertial emulation.

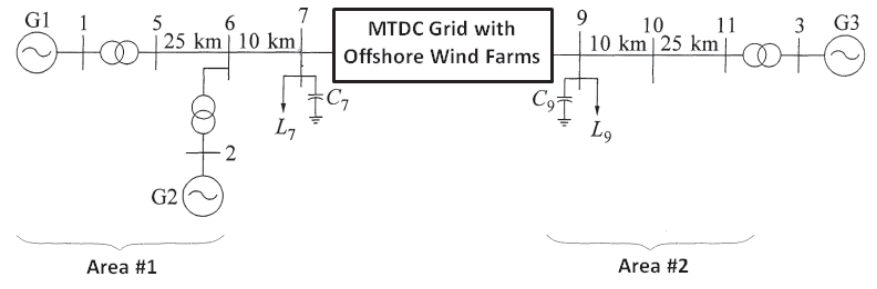

Fig. 4. Test system configuration with two asynchronous control areas (adapted from [19])

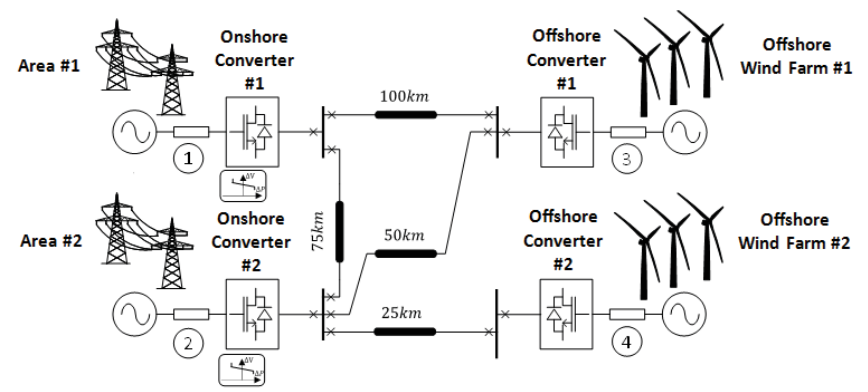

Fig. 5. Multi-terminal HVDC grid with offshore wind farms

\section{A. Analysis of the system performance for Scenario I}

As it was previously stated, this section aims on demonstrating the effectiveness of the proposed control strategy regarding the participation of MTDC system on primary frequency regulation mechanisms and on the provision of inertial emulation capabilities following a disturbance on the mainland AC grid. In this case, the offshore wind farms are set to operate at constant power and do not provide any type of regulation functionality. The disturbance that was simulated consisted in a step load increase of $400 \mathrm{MW}$ at $\mathrm{t}=20 \mathrm{~s}$ in the control Area \#2. It were also considered that the primary regulation capability in each VSC-HVDC converter connected to the AC control areas to be modified according to the parameters defined in Table I for each simulation case.

As it can be observed in Fig. 6 and Fig 7, the load increase in Area \#2 leads to a frequency deviation that is compensated by the active participation of the VSC-HVDC converter station connected to that control area. Additionally, it is possible to observe the active contribution of Area \#1 through the MTDC link, which is possible through the implementation of the primary frequency regulation functionality on both converter stations. Therefore, the proposed control functionality contributes to the active sharing of primary frequency regulation functionality as well as for the primary reserve among non- synchronous areas. From the obtained results is also possible to observe the impact resulting from the different parametrization adopted for the primary frequency regulation strategy that were defined in Table I. The possibility of having different parametrizations provides an import degree of flexibility to system operators in order to define the expected contribution from each control area as a function of the operational conditions.

TABLE I. VSC-HVDC PRIMARY FREQUENCY CONTROL PARAMETERS

\begin{tabular}{|c|c|c|c|}
\hline & $\begin{array}{c}\text { Frequency dead-band } \\
{\left[\mathbf{f}_{n}^{1} ; \mathbf{f}_{n}^{r}\right](\mathbf{H z})}\end{array}$ & $\begin{array}{c}{\left[\mathbf{f}_{\text {min }} ; \mathbf{f}_{\max }\right]} \\
(\mathbf{H z})\end{array}$ & $\begin{array}{c}{\left[\Delta \boldsymbol{P}_{\text {min }} ; \Delta \boldsymbol{P}_{\text {max }}\right]} \\
\text { (p.u.) }\end{array}$ \\
\hline Case A & {$[49.95 ; 50.05]$} & {$[48.5 ; 51.5]$} & {$[-0.5 ; 0.5]$} \\
\hline Case B & {$[49.95 ; 50.05]$} & {$[49 ; 51]$} & {$[-0.5 ; 0.5]$} \\
\hline Case $\mathrm{C}$ & {$[49.95 ; 50.05]$} & {$[49.5 ; 50.5]$} & {$[-0.5 ; 0.5]$} \\
\hline
\end{tabular}
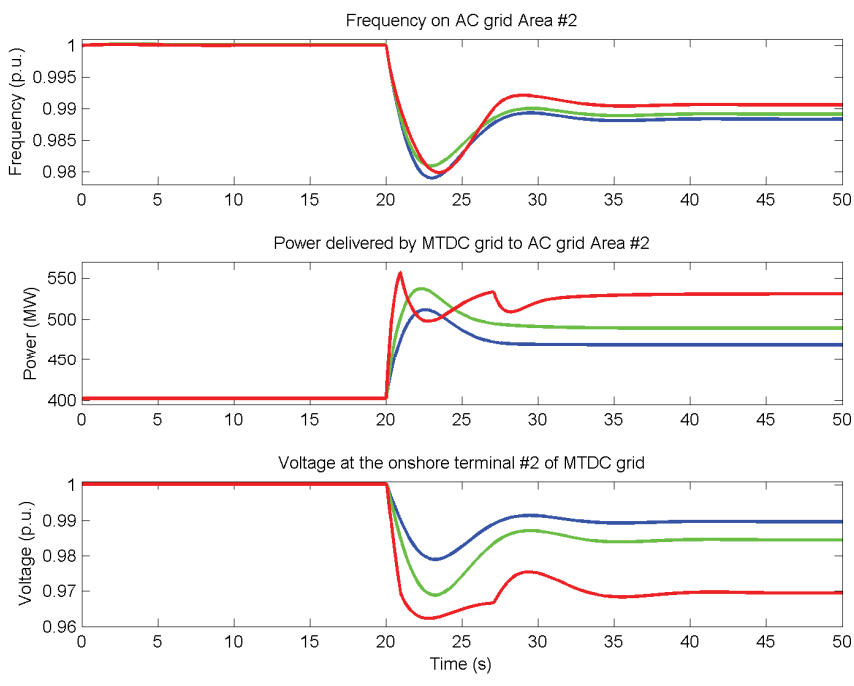

- Case A C Case B — Case C

Fig. 6. Step load increase in Area \#2: main simulation results

From the obtained results, it is also possible to observe that the active power modulation at each VSC-HVDC converter station according to the AC grid frequency influences the MTDC grid voltage profile, being it the natural communication channel to share the primary frequency regulation functionalities among the non-synchronous areas. In addition to the use of a primary frequency regulation capacity, it was also evaluated the effect resulting from the addition of inertial emulation capability to the VSC-HVDC converters connected to the AC control areas. In this case, it was selected the configuration for primary frequency control from Case B as a reference case. The benefit resulting from the inertia emulation in evidenced in Fig. 8 and Fig. 9. As it can be observed, the inertia emulation capability, represented by the active power response proportional to the time derivative of the grid frequency, provides a faster active power response, which reduces the rate of change of frequency on the area where the disturbance was considered. As a consequence of the faster active power injection into the disturbed control area, it is possible to observe a faster dynamics in the MTDC grid voltage profile in the monuments subsequent to the disturbance. Nevertheless, global system stability is assured. 

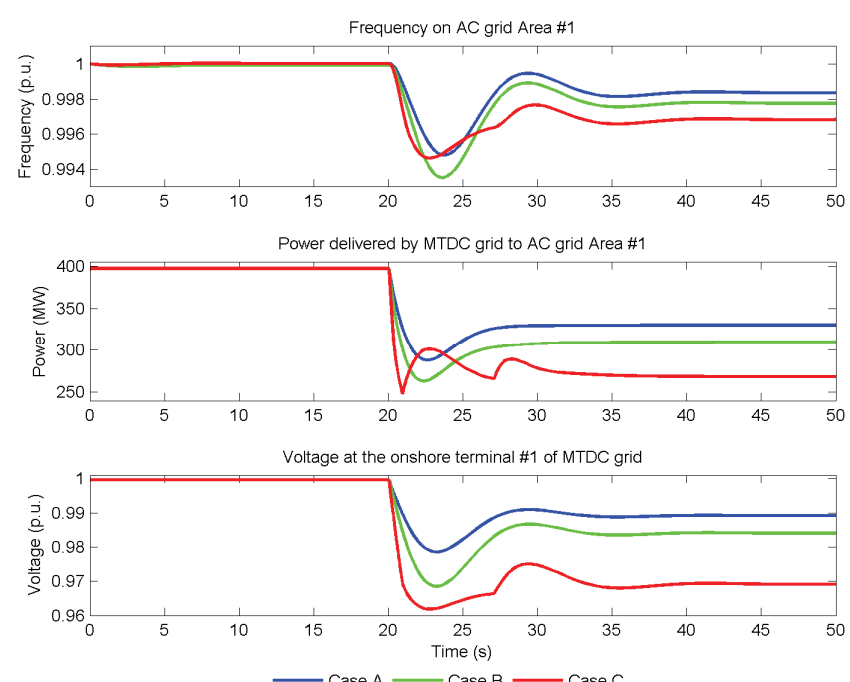

Fig. 7. Step load increase in Area \# 2: resulting impact in Area \# 1
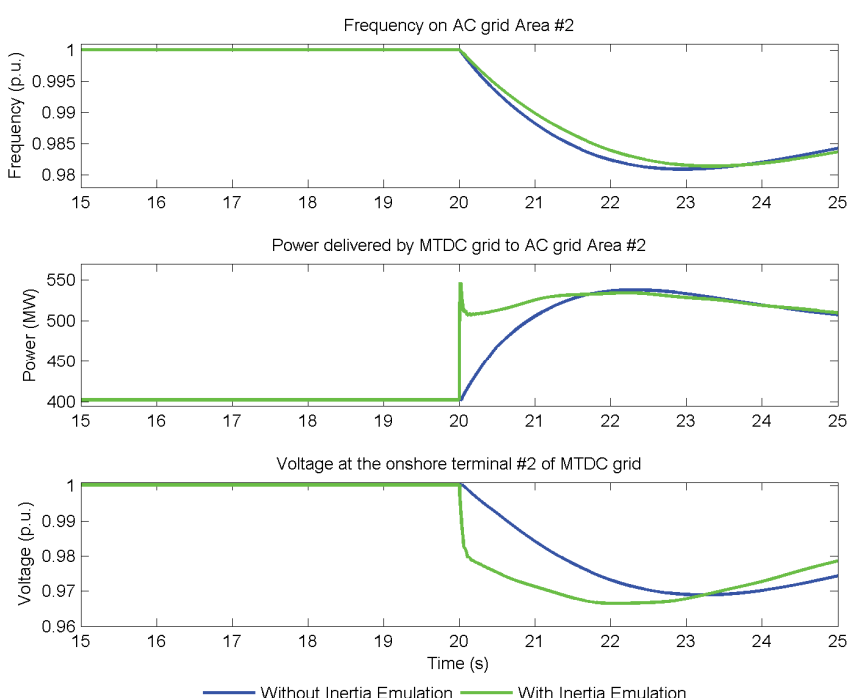

Fig. 8. Step load increase in Area \# 2: impacts resulting from the inclusion of the inertia emulation capability

\section{B. Analysis of the system performance for Scenario II}

Following the initial analysis where the offshore wind farms integrated in the MTDC system are operated with a constant power injection, it is now assumed they are able to actively participate in the primary frequency regulation service. In this case, the wind generators are assumed to be operated in a deloaded condition in order to have a primary reserve margin to be deployed following a disturbance. Therefore, as an initial simulation condition, it is assumed that both onshore converter stations have primary frequency control capability as well as the inertia emulation functionality. From the offshore wind farm side, it is assumed they have $10 \%$ active power reserve margin, being operated in the de-loaded condition. As it was previously discussed, the strategy that is proposed in order to allow the active participation of the offshore wind farms in primary frequency regulation relies on the modulation of the offshore $\mathrm{AC}$ grid frequency proportionally to the $\mathrm{DC}$ voltage variation in the associated VSC-HVDC offshore converter station (as in Fig. 3). Regarding the disturbance corresponding to the $400 \mathrm{MW}$ step load increase in the AC control Area \# 2 at $t=20 \mathrm{~s}$, the obtained results are depicted in Fig. 10 and Figure 11. As it is possible to observe, the active power injected by the onshore converter stations leads to a decrease on the DC grid voltage, which is used in order to modulate the offshore wind farms grid frequency. Thereafter, the offshore VSC-HVDC converter stations control the offshore AC grid frequency based on a droop control function in which the input is the DC voltage variation prior to the disturbance. Consequently, at the wind generator level, it is observed there is a natural response regarding the deployment of its active power reserve margin (being considered two cases, A and B, where in case A the $K_{f}$ gain defined in Fig. 3 is the double of the value considered for case B).
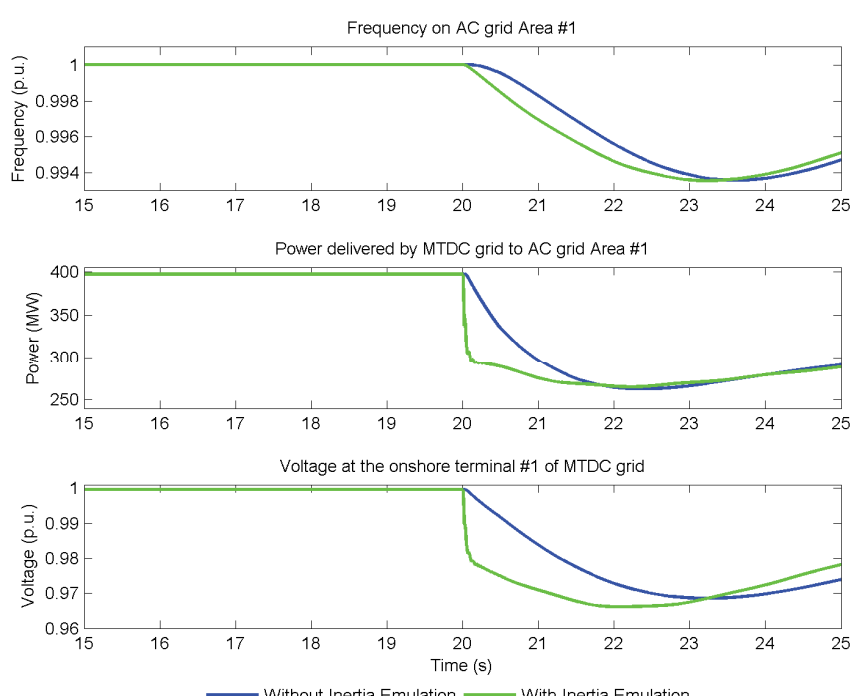

Fig. 9. Step load increase in Area \# 2: impacts in Area \#1 resulting from the inclusion of the inertia emulation capability
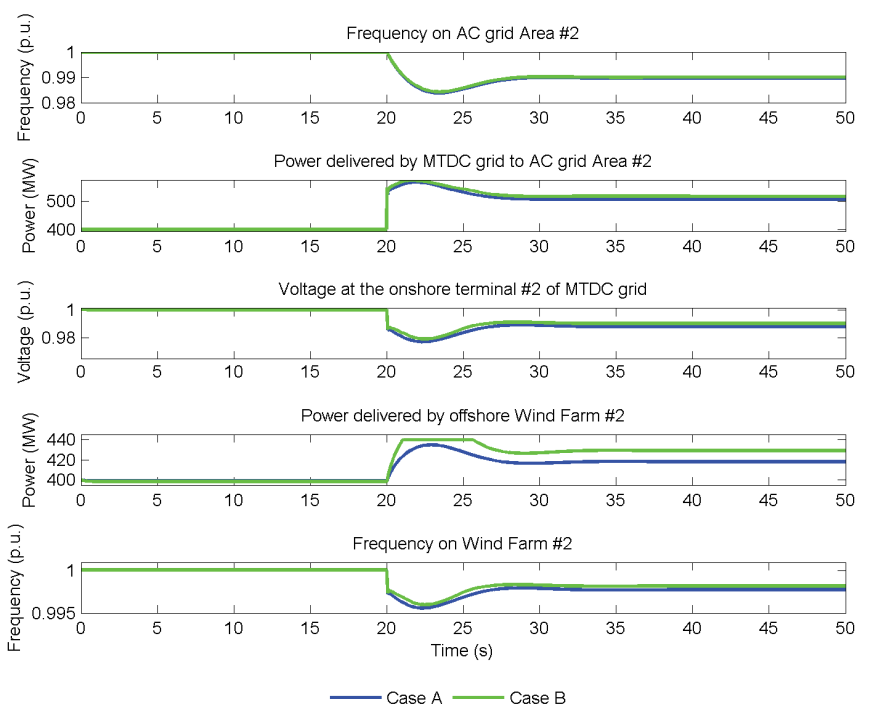

Fig. 10. Step load increase in Area \# 2: impacts resulting from the participation of offshore wind farms in primary frequency regulation 
From the obtained results, it is possible to observe that in this case, in addition to the inter-area frequency regulation support following the disturbance, there is the active contribution from the offshore wind farms in terms of the deployment of their active power reserve margin. As it was previously discussed, the deployment of active power at the wind far level is achieved thorough the exploitation of several control mechanisms that are implemented locally at the VSC-HVDC converters and at the wind generators level.
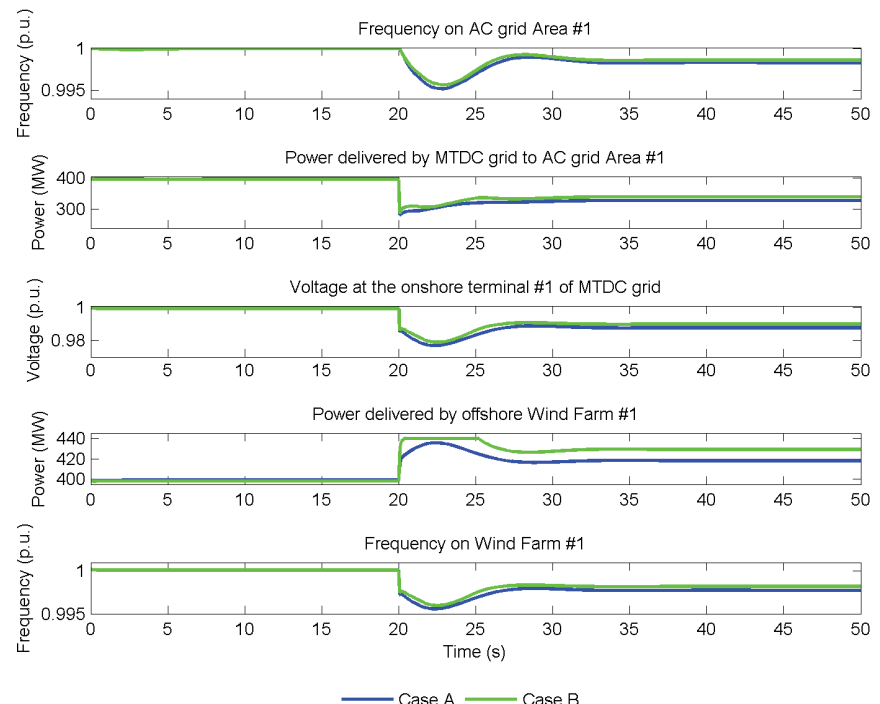

Fig. 11. Step load increase in Area \# 2: impacts in Area \#1 resulting from the participation of offshore wind farms in primary frequency regulation

Following the results described above, it is now assumed that the wind generators are complemented with an additional control loop with the purpose of providing inertial emulation capability, similarly to what happens to conventional synchronous generators connected to onshore AC grids. In order to establish a comparative analysis the same disturbance was applied in AC Area \#2. The case B from the previous simulation was taken as reference, being now compared with a scenario where wind generators contribute with inertia emulation (case $\mathrm{C}$ ), and with a case where this functionality is not considered (case B, which is equivalent to case B). The main simulation results are depicted in Fig. 12. Inertial contribution is based on a fast extraction of kinetic energy from the rotating mass of the wind generator, which is injected in the grid. Therefore, inertial emulation is able to make wind generators injecting an additional amount of power following the disturbance as a function of the time derivative of the offshore AC grid frequency. In Fig. 12 it is possible to observe that the proposed control strategy for the MTDC system is able to make wind farms contributing with inertial response following the onshore AC grid frequency disturbance. Since inertial contribution has a small duration once it is based on the release of kinetic energy form the wind turbine rotor, the response of the overall system will tend to approximate to the results presented in the previous section. However, in the moments subsequent to the disturbance it is clear that the MTDC system is able to inject more power than in the case where no inertial emulation functionalities are used. Regarding the AC control Area \#2 frequency behaviour, it is expected that the inertial emulation at the wind farm level is effective in the reduction of the frequency rate of change. This is an interesting result that can be advantageous when having Rate of Change of Frequency (ROCOF) relays in the nearby zones. Having this capability in the offshore WIND FARM can distinguish a ROCOF relay tripping / non-tripping situation in the AC network.
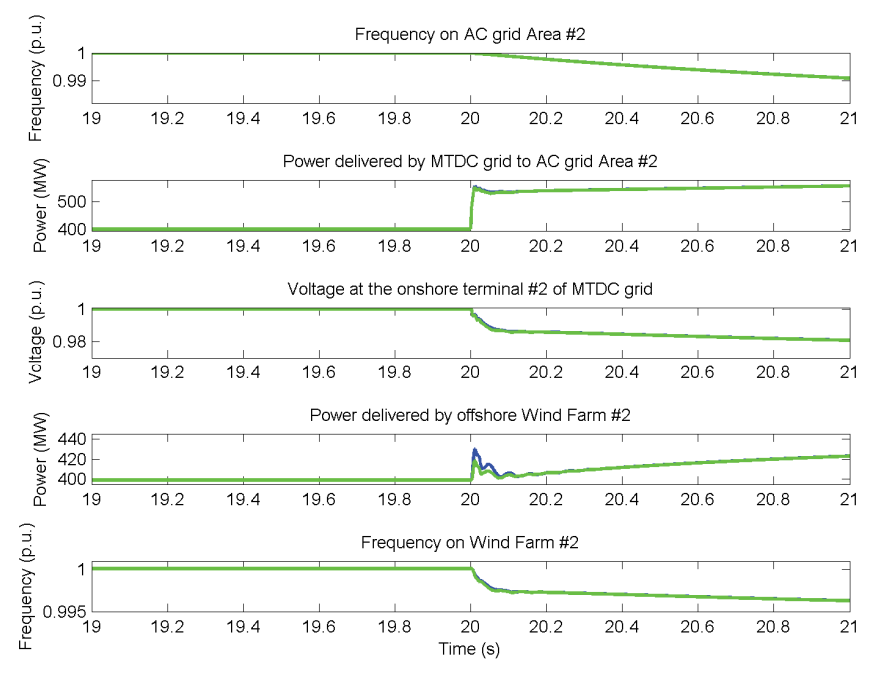

- Case C — Case D

Fig. 12. Step load increase in Area \# 2: impacts resulting from the participation of offshore wind farms with inertial emulation in primary frequency regulation

\section{CONCLUSIONS}

This paper presents an innovative approach to design operation settings of VSC-HVDC converter stations in a multiterminal DC network interconnecting non-synchronous areas an integrating offshore wind farms. The proposed control strategies endow the DC grid to actively contribute to primary frequency regulation among the nonsynchronous areas as well as to emulate inertia at the onshore converter stations, while exploiting a communication-free control solution. Additionally, it was also investigated the possibility of extending the proposed control solution in order to allow offshore wind farms to effectively contribute to support AC grid frequency, without resorting to remote communication, which could affect the reliability and efficiency of the scheme. Furthermore, results show that the control scheme achieves a significant reduction of frequency deviations in mainland systems.

In the context of a massive development of offshore power generation, the innovative controllers presented in this paper is expected to help maintaining a high-level of reliability and cost efficiency for mainland systems, in particular for islands or weak grids connected through MTDC infrastructures, as well as for scenarios characterised by a massive share of renewables. Before applying the control scheme, further work is however needed. In particular, the definition of the controller set points should be in-depth investigated. Also, the reliability of inertia and frequency control reserves with respect to disturbance in the MTDC grid should be studied. Finally, it is necessary to investigate whether the fast-response dynamics induced by the inertia and primary frequency control scheme interfere with the adopted MTDC protection schemes. 


\section{ACKNOWLEDGMENT}

This work is funded by the ERDF - European Regional Development Fund through the COMPETE Program (operational program for competitiveness) and by National Funds through the FCT - Fundação para a Ciência e a Tecnologia (Portuguese Foundation for Science and Technology) within project COMUTE-DC: Control and Operation of Offshore Multi-Terminal DC grids, PTDC/EEIEEL/2053/2012.

\section{REFERENCES}

[1] J. De Decker, and A. Woyte, "Review of the various proposals for the European offshore grid," Renewable Energy, vol. 49, pp. 58-62, 2013.

[2] European Union Project "TWENTIES." [Online]. Available: http://www.twenties-project.eu.

[3] K. Bell, D. Cirio, A. M. Denis, L. He, C. C. Liu, G. Migliavacca, C. L. Moreira, and P. Panciatici, "Economic and technical criteria for designing future offshore HVDC grids," in Innovative Smart Grid Technologies Conference Europe (ISGT Europe), 2010 IEEE PES, 2010, 11-13 Oct. 2010 .

[4] Inteligent Energy Europe. "OffshoreGrid Project." [Online]. Available: http://www.offshoregrid.eu.

[5] ENTSO-E Network Code for Requirements for Grid Connection Applicable to all Generators, ENTSO-E, Brussels, Belgium, March 2013.

[6] Requirements for Offshore Grid Connections in the Grid of TenneT TSO GmbH,TenneT TSO GmbH, Bayreuth, Germany, December 2012.

[7] R. G. de Almeida, and J. A. Peças Lopes, "Participation of doubly fed induction wind generators in system frequency regulation," IEEE Trans. on Power Systems, Vol. 22, No. 3, pp. 944-950, August 2007.

[8] S. M. Muyeen, R. Takahashi, T. Murata and J. Tamura, "A variable speed wind turbine control strategy to meet wind farm grid code requirements," IEEE Trans. on Power Systems, Vol. .25, No. 1, pp. 331-340, February 2010.

[9] Z. Miao; L. Fan; D. Osborn and S. Yuvarajan, "Wind farms with HVdc delivery in inertial response and primary frequency control," IEEE Trans. on Energy Conversion, Vol. 25, No. 4, pp. 1171-1178, December 2010.

[10] J. Morren, S. W. H. de Haan, W. L. Kling, and J. A. Ferreira, "Wind turbines emulating inertia and supporting primary frequency control," IEEE Trans. on Power Systems, Vol. 21, No. 1, pp. 433-434, February 2006.

[11] A. Teninge, C. Jecu, D. Roye, S. Bacha, J. Duval and R. Belhomme, "Contribution to frequency control through wind turbine inertial energy storage," IET Renewable Power Generation, Vol. 3, No. 3, pp. 358-370, September 2009.

[12] ENTSO-E Draft Network Code on High Voltage Direct Current Connections and DC connected Power Park Modules, ENTSO-E, Brussels, Belgium, March 2014.

[13] A. Junyent-Ferré, Y. Pipelzadeh and T. Green, "Blending HVDC-Link Energy Storage and Offshore Wind Turbine Inertia for Fast Frequency Response," IEEE Transactions on Sustainable Energy, doi: 10.1109/TSTE.2014.2360147.

[14] Z. Miao; L. Fan; D. Osborn and S. Yuvarajan, "Wind farms with HVdc delivery in inertial response and primary frequency control," IEEE Trans. on Energy Conversion, Vol. 25, No. 4, pp. 1171-1178, December 2010.

[15] S. Cole, J. Beerten and R. Belmans, "Generalized dynamic VSC MTDC model for power system stability studies," IEEE Trans. on Power Systems, Vol. 25, No. 3, pp. 1655-1662, August 2010.

[16] J. Liang, T. Jing, O. Gomis-Bellmunt, J. Ekanayake and N. Jenkins, "Operation and Control of Multiterminal HVDC Transmission for Offshore WIND FARM," Power Delivery, IEEE Transactions on, vol.pp, no.99, pp.1, 0 doi: 10.1109/TPWRD.2011.2152864.

[17] S. M. Muyeen, R. Takahashi and J. Tamura, "Operation and control of HVDC-connected offshore wind farm," IEEE Trans. on Sustainable Energy, Vol. 1, No. 1, pp. 30-37, April 2010.

[18] O. Gomis-Bellmunt, J. Liang, J. Ekanayake and N. Jenkins, "Voltagecurrent characteristics of multiterminal VSC-HVDC for offshore wind farms," Electric Power Systems Research, Vol. 81, No. 2, pp. 440-450, February 2011.

[19] P. Kundur, "Power system Stability and Control", New York, NY, USA: McGraw-Hill, 1994. 